\title{
A New Model for the Backscatter Coefficient in Nanoscale MOSFETs
}

\author{
J.-L.P.J. van der Steen ${ }^{\dagger}$, P. Palestri*, D. Esseni* and R.J.E. Hueting ${ }^{\dagger}$ \\ ${ }^{\dagger} \mathrm{MESA}^{+}$, University of Twente, 7500AE Enschede, The Netherlands \\ *DIEGM, University of Udine, Via delle Scienze 208, 33100 Udine, Italy \\ Email: j.l.p.j.vandersteen@utwente.nl
}

\begin{abstract}
In this work, we present a new model for the backscatter coefficient in nanoscale MOSFETs. The model assumes that only few backscattering events occur, which is likely to hold for devices with channel length in the order of the carrier mean free path. Both elastic and inelastic scattering mechanisms are accounted for. Moreover, the model naturally captures the effect of degeneracy. The model is compared with Monte-Carlo simulations for a broad range of channel lengths, temperatures, and electric fields, obtaining in general a very good agreement.
\end{abstract}

\section{INTRODUCTION}

In today's extremely scaled MOSFETs, the channel length is in the order of the carrier mean free path $(\lambda)$. Consequently, carriers encounter only few scattering events when moving from source to drain, commonly referred to as quasi-ballistic transport. Some of the carriers do not reach the drain since they are backscattered to the source. The so-called backscatter coefficient $(r)$ is given by the ratio of fluxes of respectively the negatively and positively directed carriers at the virtual source (VS), i.e. the top of the source/channel barrier. Modelling of $r$ is an active area of research [1]-[3] since $r$ provides an estimate of how close to the ballistic limit a device operates Since carriers experience only few scattering events, the carrier distribution will deviate significantly from the equilibrium one. To illustrate this point, Fig. 1 shows the velocity distribution along the channel of a $32 \mathrm{~nm}$ Single-Gate SOI MOSFET obtained from a Multi-Subband Monte Carlo simulator (MSMC) [4]. The device parameters are reported in [5]. Up to a distance of approximately $L_{\mathrm{kT}}$, i.e. where the voltage drop from the VS equals the thermal voltage, the positive velocity distribution essentially retains its initial Maxwellian shape, slightly displaced towards higher $v_{\mathrm{x}}$. However, as we move further along the channel towards the drain, the distribution starts to deviate significantly from a Maxwellian ('thermal') distribution. The well-known model for $r$, originally proposed in [6] and elaborated upon in [7], relates $r$ to $L_{\mathrm{kT}}$

$$
r=\frac{L_{\mathrm{kT}}(1-\beta)}{L_{\mathrm{kT}}(1-\beta)+\lambda}
$$

with $\beta=\exp \left(-L / L_{\mathrm{kT}}\right), \lambda=2 \mu K_{\mathrm{B}} T / q \nu_{\mathrm{th}}$ and $\nu_{\mathrm{th}}=$ $\sqrt{2 K_{\mathrm{B}} T / \pi m_{\mathrm{x}}}$. However, as shown in [7], (1) implicitly assumes a linear potential profile, and a thermalized carrier distribution. Furthermore, $\lambda$ is related to the low-field mobility $(\mu)$, so it is not directly linked to the individual scattering mechanisms and does not separate the effects of elastic and inelastic scattering.

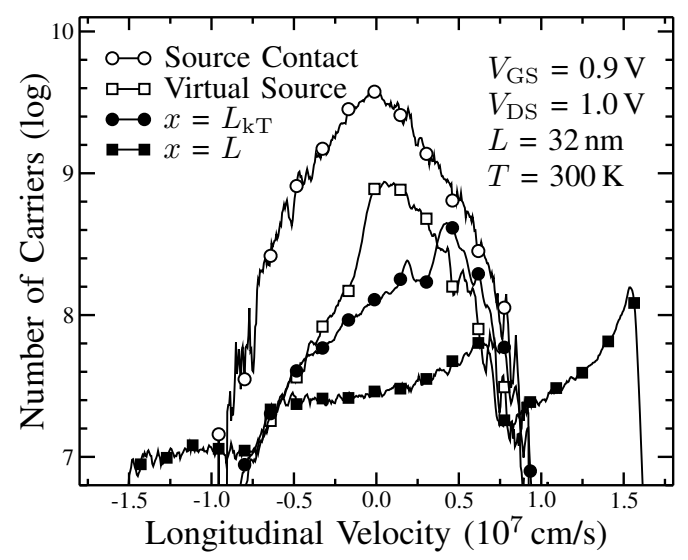

Fig. 1. Velocity distribution at several points along the channel of a $32 \mathrm{~nm}$ Single-Gate SOI MOSFET [5]: at the source contact, at the virtual source $(x=0)$, at $L_{\mathrm{kT}}$ (i.e. where the voltage drop from the virtual source equals the thermal voltage, $L_{\mathrm{kT}} \approx 5 \mathrm{~nm}$ ), and at the drain end of the channel $(L)$.

In this work, we introduce a new model for $r$ which, differently from [6], does not require the fluxes to be close to equilibrium nor does it pose any restriction on the shape of the backscattered distribution. Furthermore, this model treats elastic and inelastic scattering separately, and can handle situations with a strong carrier degeneracy (e.g. such as at the VS of MOSFETs operating in the ON-condition).

Using a simple test case, the model results are compared with values obtained from MSMC simulations [4], [8], for a wide range of electric field, channel length and temperature values.

\section{MOdEL FrAmEWORK}

Before proceeding to the derivation of the model, we briefly discuss the assumptions which are central to the model framework. First, we assume that only few scattering events occur in the channel and that, in particular, those carriers which are scattered back to the VS, have encountered on average just a single scattering event. Further, we consider a single subband with parabolic energy dispersion.

\section{A. Derivation of the Single-Scattering model}

We assume that the flux of particles $F^{+}$moving from the VS to the drain can be considered as a ballistic one, i.e. back-scattering events are very rare and do not significantly affect $F^{+}$. We denote as $I_{0}^{+}$the current associated with $F^{+}$. The energy distribution of the charges belonging to $F^{+}$is 


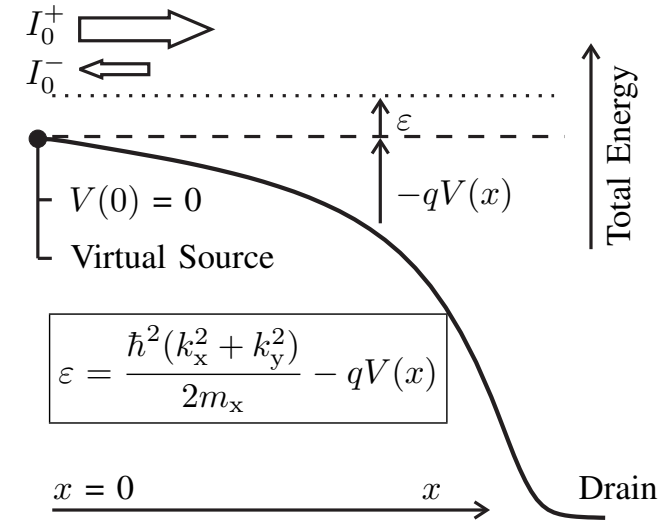

Fig. 2. Schematic of the model framework. $I_{0}^{+}$and $I_{0}^{-}$are resp. the injected ballistic flux and the backscattered flux, at the virtual source; $\varepsilon$ is the total energy and $V(x)$ is the potential.

indicated as $n^{+}(x, \varepsilon)$. The integral of $n^{+}(x, \varepsilon)$ over the total energy $\varepsilon$ gives the inversion density of carriers moving inside the channel with positive group velocity. The total energy $\varepsilon$ includes the kinetic energy and the subband energy. As sketched in Fig. 2, $\varepsilon=0$ is taken at the bottom of the subband at the VS $(x=0)$. Since we assume $F^{+}$to be ballistic, $n^{+}(x, \varepsilon)$ is null for $\varepsilon<0$ at any $x$ along the channel.

The number of carriers belonging to $F^{+}$suffering a scattering event per unit time, unit distance and unit energy is given by $n^{+}(x, \varepsilon) / \tau$, where $1 / \tau$ is the scattering rate. At this stage we assume $\tau$ not to depend on energy and assume the scattering events to be elastic and isotropic. The backscattered flux $I_{0}^{-}$ at the VS can thus be expressed in terms of $n^{+}(x, \varepsilon), \tau$, and the probability $\alpha(x, \varepsilon)$ that a carrier, once scattered, reaches the virtual source again. We write

$$
I_{0}^{-}=q \int_{0}^{L} \int_{0}^{\infty} \frac{n^{+}(x, \varepsilon)}{\tau} \alpha(x, \varepsilon) d \varepsilon d x
$$

with $L$ the channel length. The probability $\alpha(x, \varepsilon)$ that a carrier with total energy $\varepsilon$, after scattering at position $x$, has sufficient longitudinal energy to surmount the barrier and to arrive at the source, is given by [6]

$$
\alpha(x, \varepsilon)=\frac{1}{\pi} \arccos \sqrt{\frac{q V(x)}{\varepsilon+q V(x)}}
$$

We note that $\alpha(x, \varepsilon)=0.5$ at the source and that it gradually decreases towards the drain. So, even if we do not embrace any concept of $K_{\mathrm{B}} T$-layer or similar, the scattering events occurring close to the VS are the most effective in backscattering carriers and, consequently, give the main contribution to $r . n^{+}(x, \varepsilon)$ can be determined by considering that, under ballistic transport, the carrier energy distribution can be obtained following the approach in [4]. However, here we consider the total energy, rather than the longitudinal energy.
Assuming Fermi-Dirac (FD) statistics, we obtain:

$$
\begin{aligned}
F(x, \varepsilon) & =\frac{N_{0}^{+}}{K_{\mathrm{B}} T \ln [1+\exp (\eta)]} \\
\times & \frac{1}{\exp \left(\frac{\varepsilon}{K_{\mathrm{B}} T}-\eta\right)+1} \frac{2}{\pi} \arccos \sqrt{\frac{q V(x)}{\varepsilon+q V(x)}}
\end{aligned}
$$

with $N_{0}^{+}$the inversion density of carriers with positive group velocity at the VS; $\eta$ is the degeneracy level, defined as $\eta=$ $\left[E_{\mathrm{F}}-E_{0}\right] / K_{\mathrm{B}} T$ at the VS.

Since the ballistic current is $I_{0}^{+}=q N_{0}^{+} v_{\text {inj }}$ with $v_{\text {inj }}=\nu_{\text {th }}$. $\left(\mathcal{F}_{1 / 2} / \mathcal{F}_{0}\right)$ [9], $\mathcal{F}_{0}=\ln [1+\exp (\eta)]$ and $\mathcal{F}_{1 / 2}(\eta)$ the FD integral of order $1 / 2$, we can express $N_{0}^{+}$in (4) as a function of $I_{0}^{+}$and then write

$$
n^{+}(x, \varepsilon)=\gamma(x, \varepsilon) \cdot \frac{I_{0}^{+}}{q}
$$

with

$$
\begin{aligned}
\gamma(x, \varepsilon) & =\frac{1}{K_{\mathrm{B}} T \ln [1+\exp (\eta)] v_{\text {inj }}} \\
\times & \frac{1}{\exp \left(\frac{\varepsilon}{K_{\mathrm{B}} T}-\eta\right)+1} \frac{2}{\pi} \arccos \sqrt{\frac{q V(x)}{\varepsilon+q V(x)}}
\end{aligned}
$$

Note that $V(x)$ can have an arbitrary profile with $V(x) \geq 0$. Substituting (5) in (2) and assuming no injection from the drain, yields

$$
r \equiv \frac{I_{0}^{-}}{I_{0}^{+}}=\int_{0}^{L} \int_{0}^{\infty} \frac{\gamma(x, \varepsilon)}{\tau} \alpha(x, \varepsilon) d \varepsilon d x
$$

\section{B. Reduction of the Positive Flux}

In presence of non-negligible scattering, the positive flux $I^{+}(x)$ will significantly decrease along the channel due to the carriers which change momentum from positive to negative. In this section, we discuss how scattering modifies the energy distribution of this flux, denoted with $I^{+}(x, \varepsilon)$. The reduction of $I^{+}(x, \varepsilon)$ is proportional to $n^{+}(x, \varepsilon)$ and to $1 / \tau$. Since the scattering is assumed to be isotropic, the probability that a carrier's momentum is redirected towards the source equals $1 / 2$. Thus, we can write

$$
\frac{d I^{+}(x, \varepsilon)}{d x}=-\frac{q n^{+}(x, \varepsilon)}{2 \tau}=-\frac{1}{2 \tau} \frac{I^{+}(x, \varepsilon)}{v_{x}^{+}(x, \varepsilon)}
$$

where we have used $I^{+}(x, \varepsilon)=q n^{+}(x, \varepsilon) v_{x}^{+}(x, \varepsilon)$. The velocity $v_{x}^{+}(x, \varepsilon)$ is assumed to be equal to the velocity of a ballistically moving flux, which can be shown to be

$$
v_{x}^{+}(x, \varepsilon)=\sqrt{\frac{2 \varepsilon}{m_{\mathrm{x}}}} \frac{1}{\arccos \sqrt{\frac{q V(x)}{\varepsilon+q V(x)}}}
$$

Then, we find for $I^{+}(x, \varepsilon)$

$$
I^{+}(x, \varepsilon)=I^{+}(0, \varepsilon) \exp \left(-\int_{0}^{x} \frac{1}{2 \tau v_{x}^{+}\left(x^{\prime}, \varepsilon\right)} d x^{\prime}\right)
$$




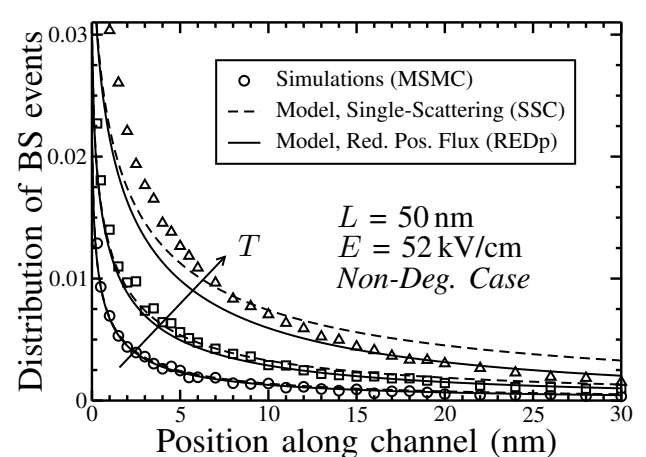

Fig. 3. Distribution of backscattering events contributing to $r$ [10]. The plot shows both the model and MSMC values for $\mathrm{T}=100-400 \mathrm{~K}$. The curves are normalized such that integrating yields $r$, the backscatter coefficient.

The carrier concentration is then given by

$$
n^{+}(x, \varepsilon)=N_{0}^{+} v_{\text {inj }} \gamma(x, \varepsilon) \exp \left(-\int_{0}^{x} \frac{1}{2 \tau v_{x}^{+}\left(x^{\prime}, \varepsilon\right)} d x^{\prime}\right)
$$

We thus see that $r$ can be obtained from (7) by replacing $\gamma(x, \varepsilon)$ with

$$
\gamma^{*}(x, \varepsilon)=\gamma(x, \varepsilon) \exp \left(-\int_{0}^{x} \frac{1}{2 \tau v_{x}^{+}\left(x^{\prime}, \varepsilon\right)} d x^{\prime}\right)
$$

\section{RESUlTS AND DiscUSSION}

In this section we compare the model results with the MSMC backscatter characteristics, for varying $L$, temperature $T$ and longitudinal field $E$. To facilitate a clear and direct comparison, we employ, unless stated otherwise, a constant accelerating field, a template material (as in [8]) and no injection from the drain. The template material features a single spherical valley $\left(m_{\mathrm{x}}=1.0 m_{0}\right)$, and $\mu=400 \mathrm{~cm}^{2} / \mathrm{Vs}$ at $300 \mathrm{~K}$, obtained by adjusting the coupling constant of acoustic phonons. Since we consider a single circular subband with parabolic energy dispersion, the scattering rate with elastic acoustic phonons, $1 / \tau$ in the model, is constant over energy and has been taken from the MSMC simulator without any adjustment in all forthcoming figures.

\section{A. Elastic Scattering}

Initially, we use Boltzmann's approximation, which in our model is naturally obtained by setting $\eta \ll 0$ in (6). The impact of degeneracy will be discussed in the second part of this section. Fig. 3 shows, for several temperatures, the distribution of carriers backscattered to the VS versus the position at which the momentum was redirected towards the source. Most scattering events which contribute to $r$ occur close to the VS. Clearly, the single-scattering model [(6)(3), labeled SSC] is able to capture the general features of the distribution produced by the MSMC. The quantitative agreement in the tail of the distribution can be improved by accounting for reduction of the positive flux [(7) with (11), REDp], particularly at high temperature and under low-field conditions, i.e. conditions which induce enhanced scattering.

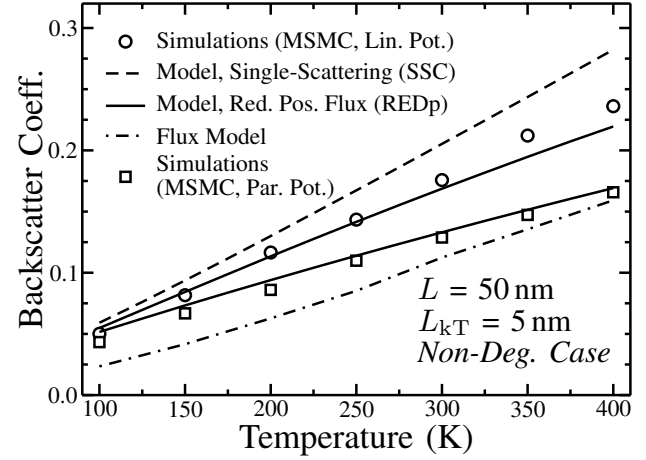

Fig. 4. $\quad r$ vs. temperature, along with the Flux model values from (1). The MSMC and REDp results are shown for a linear potential profile (corresponding to $E=52 \mathrm{kV} / \mathrm{cm}$ ) and a parabolic potential. The SSC and Flux model values are shown for the linear profile only.

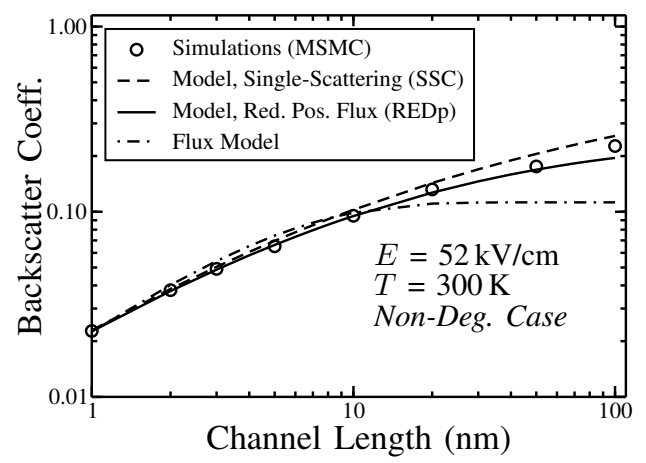

Fig. 5. $r$ vs. channel length; $E=52 \mathrm{kV} / \mathrm{cm}, \mathrm{T}=300 \mathrm{~K}$. The SSC model starts to deviate from the MSMC values for the longer channel lengths. The agreement can be improved by accounting for reduction of the positive flux.

Fig. 4 shows the values of $r$, corresponding to the curves in Fig. 3, along with the values predicted by the conventional model based on the thermal fluxes ["Flux model", (1)]. The value of $\lambda$, to be used in (1), is obtained by noting that the low-field mobility $\mu$ is related to the scattering rate $(1 / \tau$ in the SSC model) through $\mu=q \tau / m_{\mathrm{x}}$, hence $\lambda=\tau \sqrt{2 \pi K_{\mathrm{B}} T / m_{\mathrm{x}}}$. By accounting for the reduction of the positive flux, the model values essentially coincide with the MSMC results for both the linear and parabolic potential profile. The Flux model systematically underestimates $r$, which is attributed to the nonthermal nature of the negative flux [11].

Fig. 5 depicts $r$ versus $L$, showing that the SSC tracks fairly well the MSMC results; again, the agreement can be improved by accounting for the positive flux reduction. The $r$ predicted by the Flux model, instead, saturates for the longer channels. The findings from Figs. 4 and 5 are concisely summarized in Fig. 6, which shows $r$ for different values of $E$. Clearly, the entire range of $r(E)$ is captured by the REDp model. For the higher fields (corresponding to $r<0.2$ ), the singlescattering assumption of the SSC model turns out to be a good approximation, judging from the agreement in the SSC and MSMC results. Eq. (1) shows good agreement for the lowest fields only, which can be explained by noting that it assumes close to equilibrium (hence low-field) conditions.

In Figs. 3-6 we have assumed a non-degenerate electron gas. Fig. 7, instead, reports $r$ as a function of the degree of 


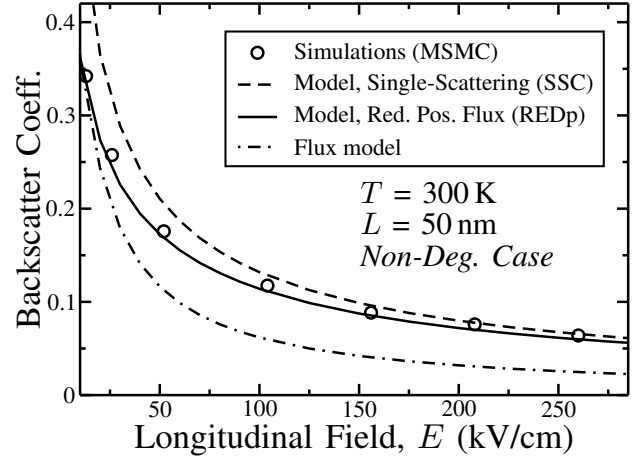

Fig. 6. $r$ vs. longitudinal field, showing the model with and without reduction of the positive flux, along with the MSMC and the flux model results.

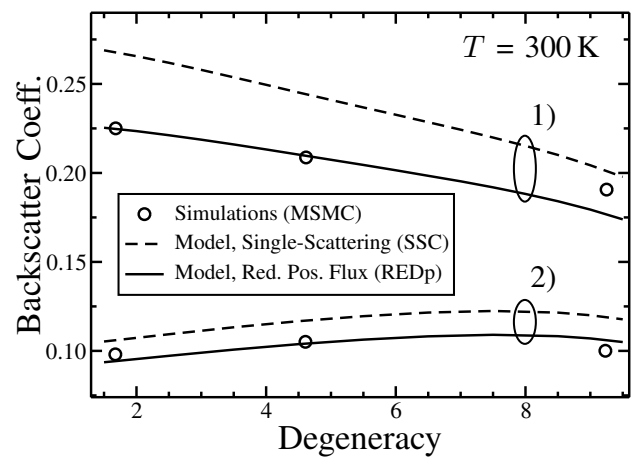

Fig. 7. $r$ for different values of the degeneracy level $\eta$, showing two sets of curves: set 1) uses $L=20 \mathrm{~nm}, E=13 \mathrm{kV} / \mathrm{cm}$; set 2) is obtained with $L=50 \mathrm{~nm}, E=156 \mathrm{kV} / \mathrm{cm}$. In either case, $r$ is found to be only weakly dependent on $\eta$.

degeneracy at the VS, for both a low [set 1)] and a highfield case [set 2)]. Although $r$ decreases [set 1)] or reaches a maximum [set 2)], the changes in absolute terms are very modest, both in the model and in the simulations.

\section{B. Inelastic Scattering}

So far, we have only considered backscattering due to elastic scattering (e.g. acoustic phonons). In order to include also optical phonon (OP) absorption and emission, (3) can be extended to

$$
\begin{gathered}
\alpha^{*}(x, \varepsilon)=\left(\frac{1}{\tau_{\mathrm{AP}}} \frac{1}{\pi} \arccos \sqrt{\frac{q V(x)}{\varepsilon+q V(x)}}\right. \\
+\frac{1}{\tau_{\mathrm{OE}}} \frac{1}{\pi} \arccos \sqrt{\frac{q V(x)}{\varepsilon-E_{\mathrm{OP}}+q V(x)}} \theta\left(\varepsilon-E_{\mathrm{OP}}\right) \\
\left.+\frac{1}{\tau_{\mathrm{OA}}} \frac{1}{\pi} \arccos \sqrt{\frac{q V(x)}{\varepsilon+E_{\mathrm{OP}}+q V(x)}}\right)
\end{gathered}
$$

in which $\tau_{\mathrm{OA}}^{-1}$ and $\tau_{\mathrm{OE}}^{-1}$ are the scattering rates of $\mathrm{OP}$ absorption and emission, respectively; $E_{\mathrm{OP}}$ is the $\mathrm{OP}$ energy, and $\theta(\mathrm{x})$ is the unit step function which ensures that OP emission can occur only if $\varepsilon \geq E_{\mathrm{OP}}$. To illustrate the impact of OP scattering, Fig. 8 depicts $r$ versus $E_{\mathrm{OP}}$. The OP coupling constant $D_{\mathrm{OP}}$ in the MSMC was increased to have significant OP scattering. $D_{\mathrm{OP}}$ used in Figs. $3-7$ was lower $\left(2 \times 10^{8} \mathrm{eV} / \mathrm{cm}\right)$, causing scattering to be dominated by acoustic rather than

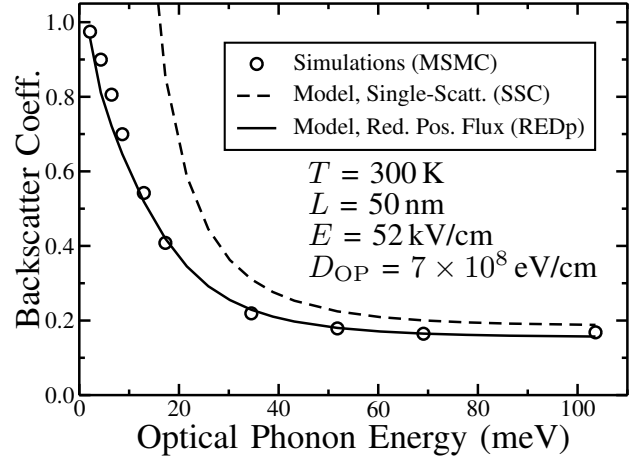

Fig. 8. $r$ vs. the optical phonon energy $E_{\mathrm{OP}}$. For each $E_{\mathrm{OP}}$, the scattering rates have been directly obtained from the scattering rates in the MSMC simulator.

optical phonons. The model values are obtained from (7) and (12). To include the positive flux reduction, (11) is modified by adding, for each scattering mechanism, an exponential term with the corresponding $\tau$. For each $E_{\mathrm{OP}}$, the values of $\tau_{\mathrm{OA}}$ and $\tau_{\mathrm{OE}}$ have been extracted from the scattering rates calculated in the MSMC simulator, which renders the model and MSMC results directly comparable.

Both the model and the MSMC predict $r$ to be constant for $E_{\mathrm{OP}}$ above a given energy, since elastic scattering becomes dominant. For smaller values of $E_{\mathrm{OP}}, r$ quickly increases due to the onset of OP emission. In fact, for very small $E_{\mathrm{OP}}$, the OP scattering rate greatly exceeds the acoustic scattering rate $(>10 \times)$. As a result, $r$ approaches unity.

\section{CONCLUSION}

In this work we present a new model for the backscatter coefficient, which is based on the assumption of only few scattering events in the channel and does not pose any restriction on the distribution of backscattered carriers. The model accounts for the effect of degeneracy and separately takes into account both elastic and inelastic scattering. Using a simple test case, the model results have been compared with MonteCarlo simulations, showing generally a very good agreement.

\section{ACKNOWLEDGMENT}

The authors would like to thank Prof. L. Selmi for his constant support and encouragement during this work.

This work was supported by NXP Semiconductors, Eindhoven, The Netherlands and in part by the ENIAC project MODERN.

\section{REFERENCES}

[1] V. Barral et al., IEEE Trans. El. Dev., vol. 56, no. 3, pp. 408-419, 2009.

[2] C. Jeong et al., IEEE Trans. El. Dev., vol. 56, no. 11, pp. 2762-2769, 2009.

[3] M. Fischetti et al., J. Comput. Electr., vol. 8, no. 2, pp. 60-77, 2009.

[4] L. Lucci et al., IEEE Trans. El. Dev., vol. 54, no. 5, pp. 1156-1164, 2007.

[5] P. Palestri et al., Solid-State Electr, vol. 53, pp. 1293-1302, 2009.

[6] M. Lundstrom et al., IEEE Trans. El. Dev., vol. 49, no. 1, pp. 133-141, 2002.

[7] R. Clerc et al., IEEE Trans. El. Dev., vol. 53, no. 7, pp. 1634-1640, 2006.

[8] P. Palestri et al., in Proc. IEDM, 2006, pp. 1-4.

[9] F. Assad et al., IEEE Trans. El. Dev., vol. 47, no. 1, pp. 232 -240, 2000.

[10] P. Palestri et al., IEEE Trans. El. Dev., vol. 52, no. 12, pp. 2727-2735, 2005.

[11] R. Clerc et al., in Proc. ULIS, 2008, pp. 125-128. 